\title{
Optimal relay coordination of an adaptive protection scheme using modified PSO algorithm.
}

\author{
ATTEYA, A.I., EL ZONKOLY, A.M. and ASHOUR, H.A.
}

(C) 2017 IEEE. Personal use of this material is permitted. Permission from IEEE must be obtained for all other uses, in any current or future media, including reprinting/republishing this material for advertising or promotional purposes, creating new collective works, for resale or redistribution to servers or lists, or reuse of any copyrighted component of this work in other works. 


\title{
Optimal Relay Coordination of an Adaptive Protection Scheme using Modified PSO Algorithm
}

\author{
Ayatte. I. Atteya, Amany. M. El Zonkoly, Hamdy. A. Ashour \\ Electrical and Control Engineering \\ Arab Academy for Science and Technology \\ Alexandria, Egypt \\ Ayatte_ibrahim@yahoo.com, amanyelz@yahoo.com, hashour@aast.edu
}

\begin{abstract}
Recently, future smart grids are described by a dominantly fluctuating character due to the power consumption change from peak to off-peak loading conditions, the operation of micro-grids in grid-connected or islanded mode and other possible network topologies resulting in an effective change in network impedances and short circuit current level. Therefore, the situation from protection sensitivity, selectivity and speed may become more and more challenging. In this paper, Adaptive protection scheme is proposed to respond to structural variations occurred in interconnected power systems. A designed software based on Modified Particle Swarm Optimization (MPSO) algorithm is suggested to solve the relay coordination problem in modern distribution networks. In this study, the 14 IEEE bus system is tested via three power system scenarios showing the effect of adding and disconnecting of DG units and the occurrence of sudden line outages on the system. The obtained results show that the proposed algorithm has achieved optimum relay settings for each existing network topology.
\end{abstract}

Index Terms-Adaptive protection scheme, Modified particle swarm optimization, overcurrent relay coordination

\section{INTRODUCTION}

Nowadays, the addition of distributed generation (DG) in medium and low voltage power networks involves the use of small-scale distributed energy resources (DERs), which can either operate in parallel to or islanded from the utility grid. Such technologies can offer numerous advantages such as higher operating efficiency, improvement of voltage profile and enhancement of network capacity, but on the other hand has a significant impact on the control, protection and reliability of the existing power system. A traditional protection system is designed to operate for radial distribution networks with unidirectional power flow. The interconnection of DG units will consequently transform the radial topology into meshed structure with bidirectional power flow affecting both the magnitude and direction of fault currents in various parts of the system. [1]. Distribution utilities are usually protected using overcurrent relays (OCRs), which are expected to operate at a fixed fault current level. Whenever the magnitude of short circuit current changes, the degree of failure of such relays to respond to new operating conditions may be increased leading to a miscoordination between the protective devices $[2]$.
Therefore, a modernized protection approach is suggested to accommodate with various changes in the behavior of short circuit current levels, introducing the concept of "Adaptive Protection scheme" [3]. Adaptive protection is actually an "online procedure in which the relay settings have to be permanently adapted to the current operating status in order to provide a selective operation for all possible network scenarios". Adaptive protection scheme could be implemented via two phases. In phase-1, the optimum setting groups are obtained using a powerful engineering algorithm for all possible network topologies then stored in the relays in an offline mode. In phase2 , a central controller detects the existing scenario based on the circuit breaker status and selects the appropriate setting groups according to the corresponding network topology [4]. In order to guarantee a fast, reliable and secure power system protection, the coordination of OCRs should be performed by adjusting two setting values: the pickup current (Ip) and the Time Dial Setting (TDS). The pickup current can be defined as the minimum current value for which the relay begins to respond. The TDS adjusts the inverse characteristics of overcurrent device, hence controlling its time delay whenever the fault current reaches a value greater than or equal to the pickup current $[5,6]$. The coordination of OCRs for future smart grids is considered as a relatively challenging task. Therefore, many intelligent optimization algorithms are used to determine optimum relay settings in modern distribution systems. In [7], the current and TDS settings are optimized using Genetic Algorithm GA for peak and off-peak loading conditions. In [8], J. Sadeh et al. has suggested a hybrid method based on GA and linear programming (LP) to solve the relay coordination problem, in which GA is applied to compute the pickup current settings for OCRs, then TDS are solved in each iteration using linear programming technique. In [9], the authors have presented an adaptive protection scheme based on Ant Colony optimization (ACO) technique to calculate OCR settings. A benchmark comparison is carried out between ACO and GA for several case studies showing that ACO has achieved better results than GA. In [10], the optimization of relay settings is performed using differential evolution (DE) algorithm and a new protection approach is investigated which utilizes an adaptive fuzzy technique to select the optimal relay settings. In [11] and [12], a 
Modified Particle Swarm Optimization (PSO) algorithm is suggested to calculate optimal relay parameters. The modification includes the initialization of PSO particles and the addition of a repair algorithm to help PSO satisfying the coordination constraints [12].

In this paper, the concept of adaptive protection scheme is explained and the suggested solution techniques are surveyed in section I. Problem formulation and constraints are stated in section II. The difference between the typical PSO and the proposed Modified PSO algorithm is also highlighted and included in section III. The 14 IEEE bus system is tested for different case studies and results are reported in section IV showing the effectiveness of the proposed approach.

\section{PROBLEM FORMULATION}

The coordination of OCRs is formulated as a Nonlinear Optimization Problem (NLP) since the relay operating time is a nonlinear function of $\mathrm{I}_{\mathrm{p}}$ in accordance with IEC 60255 [13], as expressed in equation (1):

$$
t_{o p}=\operatorname{TDS} \frac{\mathrm{A}}{\left(I_{F} / I_{p}\right)^{B}-1}
$$

Where $\left(\mathrm{t}_{\mathrm{op}}\right)$ is the relay operating time, (TDS) is the relay time dial setting, $\left(\mathrm{I}_{\mathrm{p}}\right)$ is the pickup current setting, $\left(\mathrm{I}_{\mathrm{f}}\right)$ is the fault current, (A) is the constant for relay characteristics, and (B) is the constant representing inverse time type.

\section{A. Objective Function}

In the relay coordination problem, the main objegtive function is to minimize the summation of operating times of all relays, where pickup current and TDS are considered as the decision variables. The general form of objective function is expressed as follows:

$$
\min \sum_{k=1}^{n}\left(t_{o p . k}\right)
$$

Where, $\mathrm{n}$ is the number of relays in the system and $\left(\mathrm{t}_{\mathrm{op}, \mathrm{k}}\right)$ is the operating time of the relay $R_{k}$.

\section{B. Constraints}

The objective function defined above is subjected to the following set of constraints.

\section{Selectivity Constraint:}

The requirement of selectivity states that when a fault occurs, only the primary relay should operate to trip the fault. If the main relay fails to operate, the backup relay should clear the fault after a pre-specified delay time known as coordination time interval (CTI), normally set between $0.2 \mathrm{~s}$ and $0.5 \mathrm{~s}$. In order to satisfy such requirement, the following constraint must be added:

$$
T_{\text {backup }}-T_{\text {main }} \leq C T I
$$

Where, $\mathrm{T}_{\text {main }}$ and $\mathrm{T}_{\text {backup }}$ are the main and backup operation time, respectively. CTI is the coordination time interval between primary and backup relays.

\section{Limit Constraints:}

There is always a range for each relay setting, from which feasible solutions are encountered. Therefore, other constraints should be considered on the limits of relay parameters, including TDS and $I_{p}$ settings, that can be expressed in the following equations:

$$
\begin{gathered}
T D S_{\min } \leq T D S \leq T D S_{\max } \\
I_{p \min } \leq I_{p} \leq I_{p \max }
\end{gathered}
$$

Where TDS min and TDS max, $\mathrm{I}_{\mathrm{p} \text { min }}$ and $\mathrm{I}_{\mathrm{p} \max }$, are the minimum and maximum limits of Time Dial Setting (TDS), and pickup current $\left(\mathrm{I}_{\mathrm{p}}\right)$ settings, respectively.

\section{PROPOSED PSO ALGORITHM}

\section{A. Typical PSO Algorithm}

PSO developed by J.Kennedy and R.Eberhart in 1995 is a population based optimization algorithm inspired by the ability of a group of swarming animals such as birds or fishes to work as a whole in a given area searching for corn. This seeking behavior is validated through equations in a real valued search space. Particles move through the search space adjusting their position and their velocities according to equations (6) and (7) based on their own experience and to their neighboring particles experience in order to find the optimal solution.

$$
\begin{aligned}
& v_{i}^{k+1}=w \cdot v_{i}^{k}+c_{1} \cdot \operatorname{rand}_{1} \cdot\left(P_{\text {best } i}-X_{i}\right)+ \\
& c_{2} \cdot \operatorname{rand}_{2} \cdot\left(G_{\text {best } i}-X_{i}\right) \\
& X_{i}^{k+1}=X_{i}^{k}+v_{i}^{k+1} \\
& w=\frac{\left(w_{\max }-w_{\min }\right)}{i t e r_{\max }} * \text { iter }
\end{aligned}
$$

Where:

$w \quad$ The inertia weight of the particle that can be calculated using equation (8), where $w_{\max }$ is $0.9, w_{\min }$ is 0.4 .

iter $_{\max }$ The maximum number of iterations

iter The current iteration.

$v_{i}^{k} \quad$ The velocity of particle (i) for iteration (k)

$c_{1} \cdot c_{2} \quad$ Acceleration rates that pull each particle towards $\mathrm{P}_{\text {best }}$ and $\mathrm{G}_{\text {best }}$ positions

rand $_{1} \cdot$ rand $_{2} \quad$ Random numbers from 0 to 1

$P_{\text {best }} \quad$ Best position achieved by particle (i)

$G_{\text {best }} \quad$ Best position achieved by all particles in the swarm

$X_{i} \quad$ Position of particle (i)

\section{B. Modified PSO Algorithm MPSO}

In standard form of PSO, the initial positions are randomly generated in the entire swarm. This by consequence will not ensure that all the initial particles are encountered from feasible solution. In the proposed Modified PSO, the initial solutions are optimized based on a linear programming technique known as the "Interior-point Method". This is performed by selecting the pickup current settings randomly between minimum and 
maximum boundaries, thus the problem becomes linear, and the TDS settings are then computed using the linear programming subroutine. Another modification concerning the updating process is added in order to maintain all particles in feasible solution. In each iteration, particles are updated one after another in the entire swarm using (6), then each one will be subjected to constraint check including limitation and selectivity constraint. If the resultant population is still within the feasible search space, then accept the updated position, otherwise, particle backs to its previous position [11]. The proposed algorithm steps are illustrated in Fig. 1.

\section{TEST CASE SYSTEM}

The IEEE 14-bus system is used to evaluate the performance of the suggested MPSO algorithm. The system consists of 30 phase relays, and 5 synchronous machines located at buses $1,2,3,8,6$ as illustrated in Fig. 2. The machines located at buses 1 and 2 are used as generation units G1 and G2. G1 is considered as the main grid while $\mathrm{G} 2$ is used as a distributed generation source. The three others remaining are considered as synchronous compensators used only for reactive power support [15]. All relays are assumed to be overcurrent relays with IEC standard inverse type characteristic curves. The network is tested via three power system scenarios as illustrated in Table 1, to validate the concept of adaptive protection scheme. For each scenario, a load flow and short circuit analysis should be performed in order to achieve optimum relay setting using the suggested optimization algorithm. A deep explanation for each power system scenario will be included in next sections.

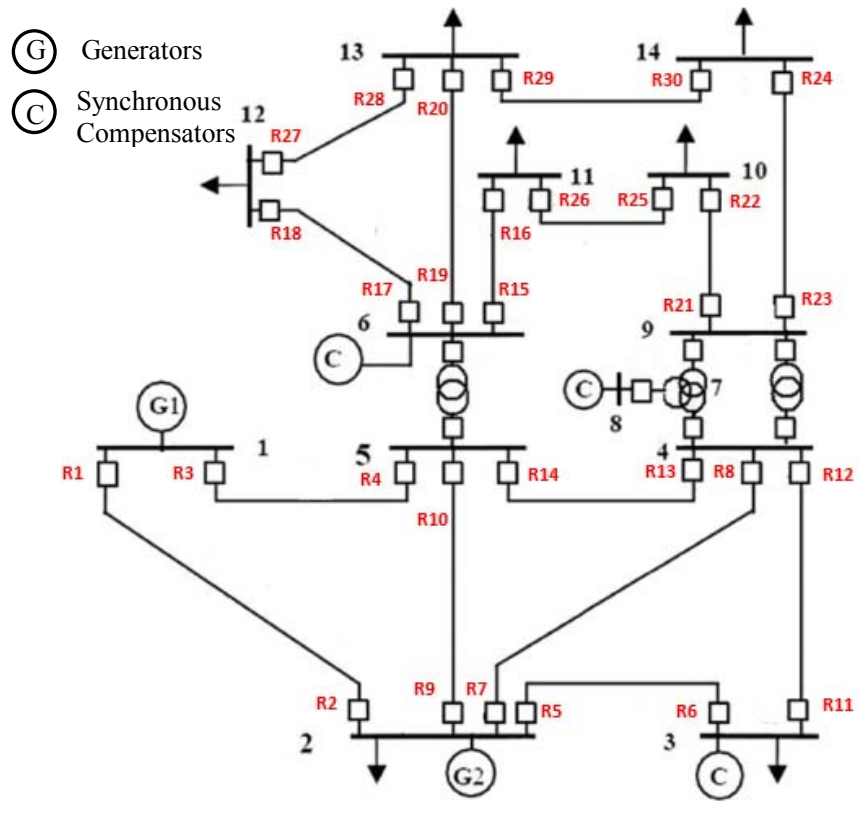

Fig. 2. The IEEE 14 bus test system

TABLE I. PROPOSAL FOR POWER SYSTEM SCENARIOS

\begin{tabular}{|lllll|}
\hline Power System Scenario & G1 & G2 & $\begin{array}{l}\text { Line } \\
\text { 1--2 }\end{array}$ & $\begin{array}{l}\text { Line } \\
\text { 2--5 }\end{array}$ \\
\hline Scenario 1 & ON & ON & ON & ON \\
\hline Scenario 2 & ON & OFF & ON & ON \\
\hline Scenario 3 - Case 1 & ON & ON & OFF & ON \\
\hline Scenario 3 - Case 2 & ON & ON & ON & OFF \\
\hline
\end{tabular}

\section{START}

$\downarrow$

Perform load flow and short circuit analysis

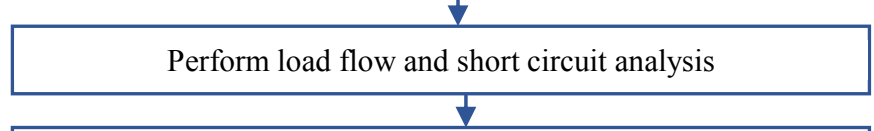

Apply the interior-point Method to select the initial positions

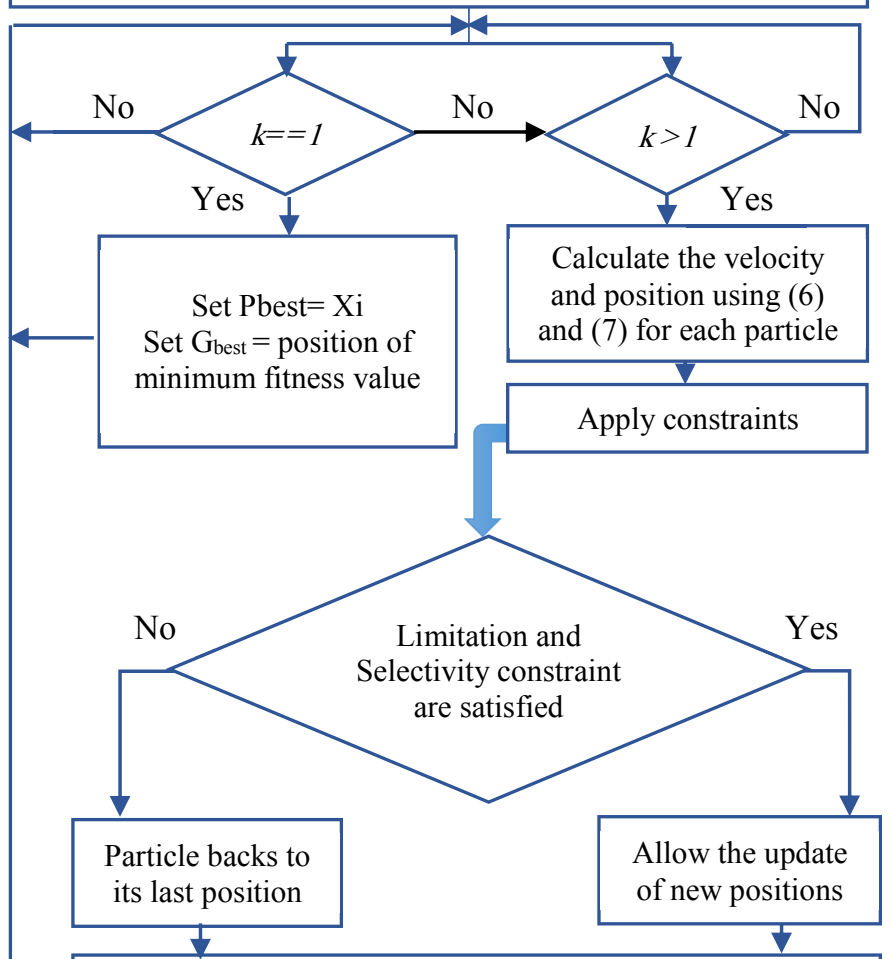

Calculate the fitness function for each particle using (2)
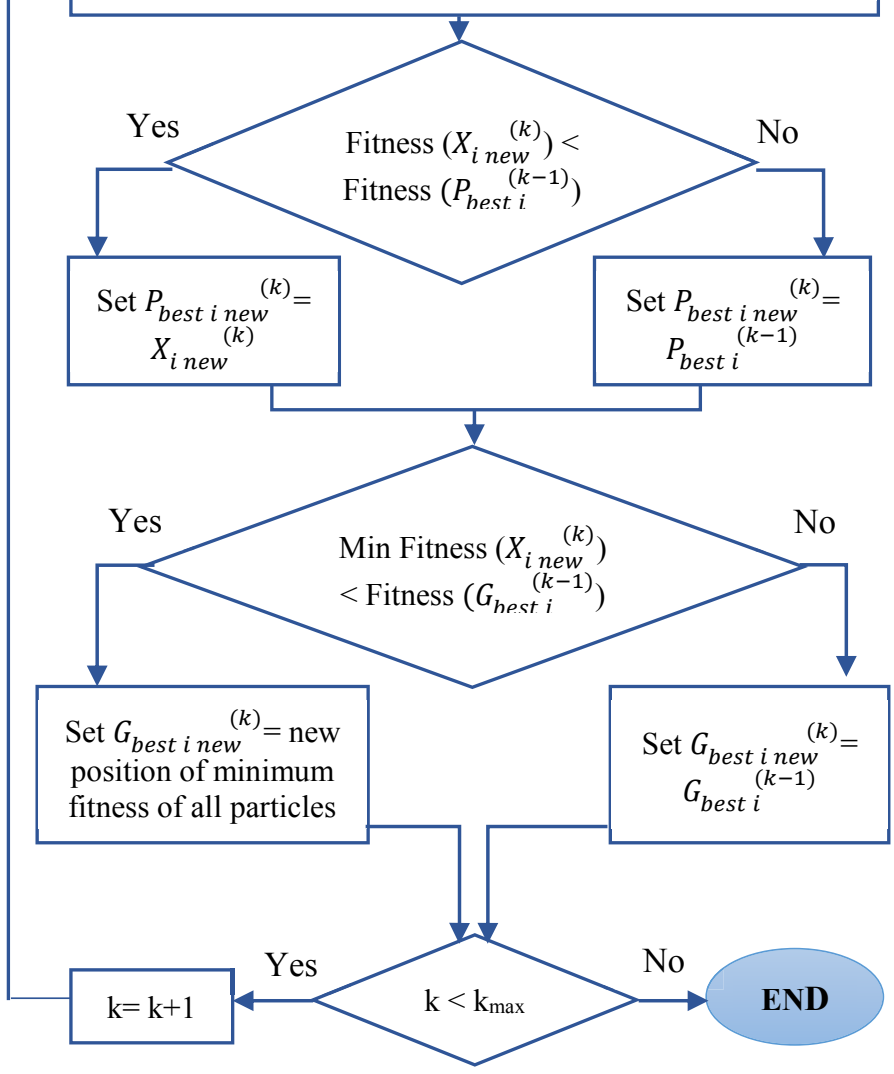

Fig. 1. The Suggested MPSO Flowchart 


\section{A. Power System Scenario 1}

The corresponding scenario represents the normal network condition when both generation units G1, G2 are operated in parallel to supply the corresponding loads. G1 is considered as the main grid, while G2 is used as a distributed generation (DG) unit operating in grid-connected mode. The determination of optimum relay setting groups can be performed using the following steps:

a. Identifying the primary and backup relay pairs for the system.

b. Performing a short circuit analysis to calculate the maximum short circuit current located at the Near-End of each relay.

c. Calculating the maximum and minimum boundaries for the pickup current setting. The upper and lower limits of current setting are considered as the full load current and the minimum short circuit current passing through the relay, respectively. Therefore, a load flow analysis is carried out to compute the relays full load currents, while the minimum short circuit current is estimated by assuming a phase-phase fault occurring at the far-end of each circuit breaker.

d. Input the calculated data entry in $(a, b, c)$ to the suggested MPSO algorithm then calculate the optimized relay settings according to the illustrated flowchart in fig.1.

The pickup current $\left(\mathrm{I}_{\mathrm{p}}\right)$ and Time Dial settings (TDS) for all the overcurrent relays are optimized using MPSO algorithm based on a swarm size of 50, and the time settings are selected between maximum and minimum bounds of 0.1 and $1.1 \mathrm{~s}$ respectively. The obtained results for Ip and TDS are listed and given in Table II.

TABLE II. TDS AND PICKUP CURRENT RELAY SETTINGS FOR POWER SYSTEM SCENARIO 1

\begin{tabular}{|l|l|l|l|l|l|}
\hline $\begin{array}{l}\text { Relay } \\
\text { No. }\end{array}$ & TDS & Ip [A] & $\begin{array}{l}\text { Relay } \\
\text { No. }\end{array}$ & TDS & Ip [A] \\
\hline R1 & 0.1 & 1965.4 & R16 & 0.1 & 8.7 \\
R2 & 0.1134 & 1805.9 & R17 & 0.1 & 77.4 \\
R3 & 0.1001 & 547.7 & R18 & 0.1 & 6.3 \\
R4 & 0.1 & 493.7 & R19 & 0.1 & 39.4 \\
R5 & 0.1 & 590.0 & R20 & 0.1 & 24.2 \\
R6 & 0.1 & 454.9 & R21 & 0.1 & 26.6 \\
R7 & 0.1412 & 522.8 & R22 & 0.1001 & 34.9 \\
R8 & 0.1 & 292.7 & R23 & 0.1001 & 44.2 \\
R9 & 0.162 & 496.7 & R24 & 0.1001 & 24.4 \\
R10 & 0.1 & 339.0 & R25 & 0.1 & 26.3 \\
R11 & 0.1054 & 312.9 & R26 & 0.1342 & 17.1 \\
R12 & 0.1026 & 318.4 & R27 & 0.1517 & 4.8 \\
R13 & 0.1 & 550.5 & R28 & 0.1 & 41.3 \\
R14 & 0.1001 & 356.6 & R29 & 0.1061 & 34.2 \\
R15 & 0.1236 & 116.4 & R30 & 0.1 & 27.6 \\
\hline
\end{tabular}

Fig.3 shows the convergence of the fitness function for the power system scenario 1, which illustrates that MPSO reaches the best solution after approximately 100 iterations. It should be noted that the convergence of fitness function shows the response of PSO algorithm after the optimization of initial generation for a population size of 50 particles using Interiorpoint-method that reaches approximately $7.9 \mathrm{sec}$ total operating time of all relays as best solution for the initial condition, then minimized to $7.2569 \mathrm{sec}$ after 100 iterations of MPSO algorithm. The coordination time intervals between primary and backup relay pairs are also presented in Fig.4. It should be noted that all the coordination intervals points are maintained between 0 and $0.5 \mathrm{sec}$ which means that MPSO has reached the minimum operating time of all relays while satisfying no more than the predefined selectivity constraint for relay coordination problem.

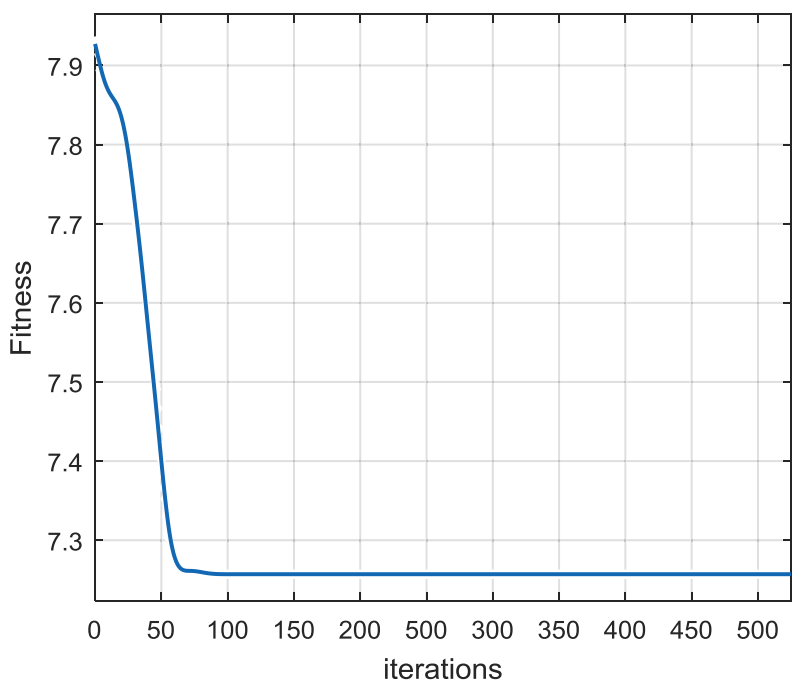

Fig.3: The convergence of proposed fitness function

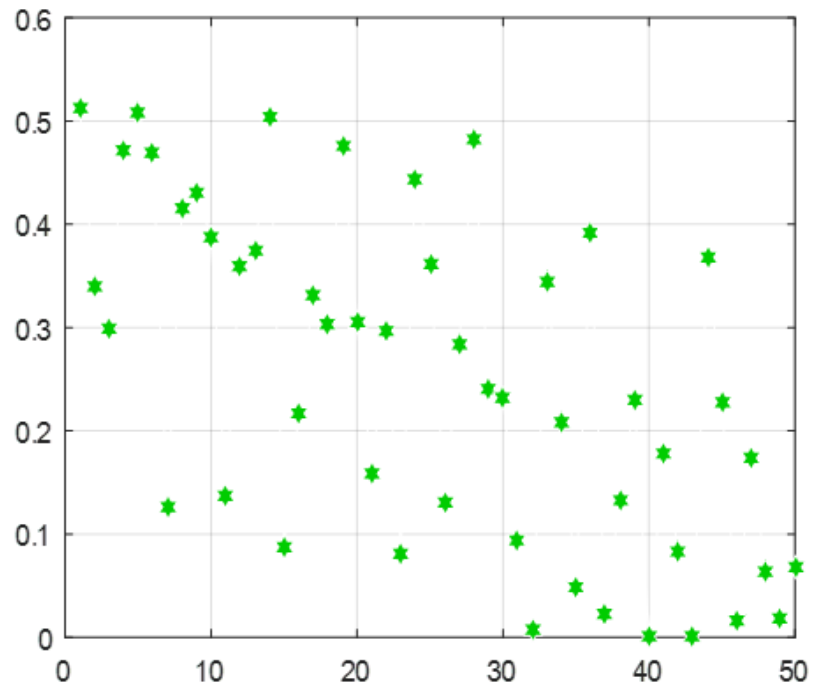

Fig.4: The coordination time interval points for all $\mathrm{P} / \mathrm{B}$ relay pairs of power system scenario 1 maintained between 0 and $0.5 \mathrm{~s}$. 


\section{B. Power System Scenario 2}

This scenario describes the operation of distributed generation unit G2 in "OFF-grid" mode. In this topology, G2 is assumed to be disconnected from the system, and only the utility grid is used to supply the whole network. This will result in a significant change in fault current level and hence may affect the selectivity of protective devices. Table III indicate the miscoordination occurred between relay pairs R2 and R6, R2 and R8, R2 and R10, R3 and R2, R22 and R26 respectively, when testing the relay settings calculated in scenario 1 on scenario 2 , returning negative coordination intervals. The results of new appropriate relay settings optimized for the second power system scenario are reported in table IV.

TABLE III. MISCOORDINATION SUMMARY OF RELAY PAIRS

\begin{tabular}{|c|c|c|}
\hline \multicolumn{2}{|c|}{ Malfunctioning P/ B relays } & CTI \\
\hline R2 & R10 & -0.75 \\
\hline R2 & R8 & -0.79 \\
\hline R2 & R6 & -0.61 \\
\hline R3 & R2 & -14.1 \\
\hline R22 & R26 & -0.01 \\
\hline
\end{tabular}

\section{Power System Scenario 3}

The third scenario describes the system in case that a "single line outage" occurs. Actually, line outages could occur by means of protection issues or maintenance purposes. Two cases of single-lines are selected and tested in order to demonstrate the effectiveness of adaptive protection technique.

- Case 1: describes the system when line 1-2 is disconnected.

- Case 2: describes the system when line 2-5 is disconnected

For each case, the upper and lower limits for relay pairs, and the short circuit currents for near and far end faults are recalculated and new relay settings are optimized to adapt the change in each test case. The computed relay settings for the two case studies are reported and given in Table VI. The convergence of fitness function for power system scenario 3 cases combined with the fitness function of scenario 2 is illustrated in Fig. 5, showing that

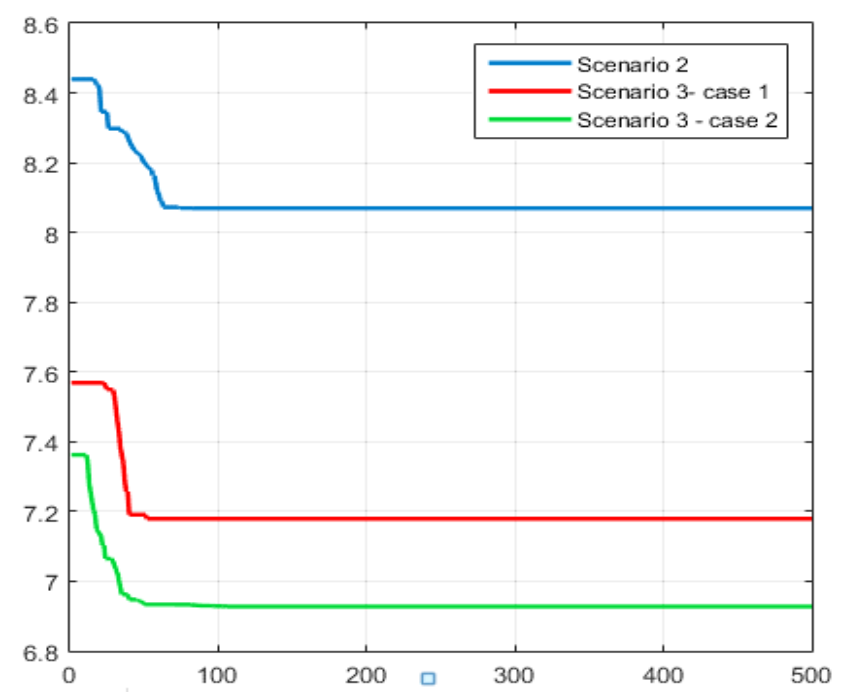

Fig.5: The combined convergence of fitness functions for power system scenarios 2 and 3 .
MPSO has achieved minimum operating time for all relays in each scenario.

TABLE IV. TDS AND PICKUP CURRENT SETTINGS SCENARIO 2 AND SCENARIO 3

\begin{tabular}{|l|l|l|l|l|l|l|}
\hline \multirow{2}{*}{$\begin{array}{l}\text { Relay } \\
\text { No. }\end{array}$} & \multirow{2}{*}{$\begin{array}{l}\text { Power System } \\
\text { Scenario 2 }\end{array}$} & \multicolumn{3}{l|}{ Power system scenario 3 } \\
\cline { 2 - 7 } & & Case 1 & \multicolumn{2}{l|}{ Case 2 } \\
\cline { 4 - 7 } & TDS & Ip [A] & TDS & Ip [A] & TDS & Ip [A] \\
\hline R1 & 0.1328 & 1595 & -- & -- & 0.1 & 1612.44 \\
R2 & 0.1008 & 498.14 & -- & -- & 0.1 & 849.139 \\
R3 & 0.1008 & 602.11 & 0.1 & 803.11 & 0.1 & 554.029 \\
R4 & 0.1005 & 283.85 & 0.1 & 473.50 & 0.1002 & 372.817 \\
R5 & 0.1 & 462.50 & 0.1 & 488.48 & 0.1 & 645.203 \\
R6 & 0.1 & 466.08 & 0.1 & 336.73 & 0.1 & 375.508 \\
R7 & 0.1001 & 438.42 & 0.1553 & 322.69 & 0.1001 & 624.696 \\
R8 & 0.1 & 279.79 & 0.1004 & 313.54 & 0.1 & 429.065 \\
R9 & 0.1220 & 363.93 & 0.1002 & 511.99 & -- & -- \\
R10 & 0.1004 & 326.90 & 0.1006 & 418.08 & -- & -- \\
R11 & 0.1013 & 343.99 & 0.1 & 479.43 & 0.1001 & 518.908 \\
R12 & 0.1008 & 289.37 & 0.1001 & 491.89 & 0.1001 & 480.105 \\
R13 & 0.1006 & 515.55 & 0.1 & 1155.68 & 0.1 & 299.238 \\
R14 & 0.1006 & 648.58 & 0.1002 & 874.09 & 0.1 & 408.657 \\
R15 & 0.15 & 64.98 & 0.1 & 130.89 & 0.1 & 73.3514 \\
R16 & 0.1005 & 28.23 & 0.1005 & 18.85 & 0.1 & 16.967 \\
R17 & 0.1 & 37.11 & 0.1 & 37.39 & 0.1001 & 14.44 \\
R18 & 0.1002 & 5.98 & 0.1 & 6.346 & 0.1 & 6.037 \\
R19 & 0.1413 & 121.00 & 0.1007 & 68.06 & 0.1 & 117.76 \\
R20 & 0.1009 & 21.96 & 0.1 & 24.59 & 0.1001 & 20.944 \\
R21 & 0.1018 & 81.02 & 0.1 & 93.43 & 0.1001 & 69.398 \\
R22 & 0.1002 & 30.57 & 0.1024 & 18.07 & 0.1 & 19.19 \\
R23 & 0.1008 & 32.55 & 0.1002 & 85.80 & 0.1005 & 76.27 \\
R24 & 0.1 & 33.76 & 0.1 & 17.45 & 0.1 & 34.74 \\
R25 & 0.1 & 29.48 & 0.1 & 29.56 & 0.1 & 34.30 \\
R26 & 0.106 & 27.59 & 0.1014 & 25.69 & 0.2201 & 2.5056 \\
R27 & 0.1 & 35.66 & 0.1010 & 12.25 & 0.1536 & 2.915 \\
R28 & 0.1041 & 5.212 & 0.1002 & 25.50 & 0.1 & 56.07 \\
R29 & 0.1998 & 9.311 & 0.1003 & 48.10 & 0.1006 & 46.39 \\
R30 & 0.1 & 12.94 & 0.1 & 27.19 & 0.1604 & 5.96 \\
& & & & & & \\
\hline
\end{tabular}

\section{CONCLUSION}

In this paper, the MPSO algorithm was proposed to solve the relay coordination problem. The modification added to the typical PSO technique helped to hold all particles in feasible solution. The 14 IEEE bus network has been tested for different power system topologies to validate the concept of adaptive protection scheme. The selected case studies have introduced the effect of DGs penetration and disconnection as well as the line outage occurrence on the distribution networks. The obtained results have demonstrated the effectiveness of the suggested approach in achieving optimum relay setting groups according to each network topology minimizing the total operation time while satisfying the selectivity constraints among all protective devices.

\section{REFERENCES}

[1] S. S. Venkata, D. Wilson, J. Ren and M. Miller " Advanced and adaptive protection for active distribution grid ", 22th International Conference on Electricity Distribution (CIRED), Stockholm, June, 2013, IEEE, pp. 1-4. 
[2] M. Singh, "Protection coordination in grid-connected and islanded modes of micro-grid operations", Innovative Smart Grid Technologies Asia (ISGT ASIA), 2013, IEEE, pp.1-6.

[3] S. Voima, H. Laaksonen, and K. Kauhaniemi, "Adaptive protection scheme for smart grids", $12^{\text {th }}$ IET International Conference on Developments in Power System Protection (DPSP), 2014, IEEE, pp. 1-6.

[4] Vasileios. A. Papaspiliotopoulos, George N. Korres, and Nikos D. Hatziargyriou, "Protection coordination in modern distribution grids integrating optimization techniques with adaptive relay setting", IEEE Power Tech conference, Eindhoven, 2015, pp. 16.

[5] M. N. Alam, B. Das and V. Pant, "A comparative study of metaheuristic optimization approaches for directional overcurrent relays coordination", Electrical Power System Research, Vol.128, 2015, Elsevier, pp. 39-52.

[6] Z. Moravej, M. Jazaeri, and M. Gholamzadeh, "Optimal coordination of distance and over-current relays in series compensated systems based on MAPSO", Energy Conversion and Management, Vol.56, 2012, Elsevier, pp. 140-151.

[7] Chao-Rong Chen and Cheng-Hung Lee, "Adaptive overcurrent relay coordination for off-peak loading in interconnected power system", Electrical Power and Energy Systems, Vol.63, 2014, Elsevier, pp.140-144.

[8] Abbas S. Noghabi and J. Sadeh, "Considering different network topologies in optimal overcurrent relay coordination using a hybrid GA", IEEE Transactions on Power Delivery, Vol.24, October, 2009, pp. 1857-1863.

[9] M. Yen Shih, Carlos A. Salazar and Arturo C. Enríquez "Adaptive directional overcurrent relay coordination using ant colony optimization", IET Generation, Transmission and Distribution, Vol.9, June, 2015, pp.2040-2049.

[10] M. Singh and V. Telukunta, "Adaptive overcurrent relay coordination algorithm for changing short circuit fault levels", Innovative Smart Grid Technologies Asia (ISGT ASIA), 2015, IEEE, pp. 1-6.

[11] H.H. Zeineldin *, E.F. El-Saadany, M.M.A. Salama, "Optimal coordination of overcurrent relays using a modified particle swarm optimization", Electric Power Systems Research, Vol.76, 2006, Elsevier, pp. 988-995.

[12] Mohamed M. Mansour, Said F. Mekhamer, and Nehad El-Sherif El-Kharbawe, "A Modified Particle Swarm Optimizer for the coordination of directional overcurrent relays", IEEE Transactions on Power Delivery, Vol.22, July, 2007, pp. 14001410.

[13] Hebatallah. M. Sharaf, H.H. Zeineldin, Doaa K. Ibrahim and Essam A. EL-Zahab, "A proposed coordination strategy for meshed distribution systems with DG considering user-defined characteristics of directional inverse time overcurrent relays", Electrical Power and Energy Systems, Vol.65, 2015, Elsevier, pp.49-58.

[14] J. Kennedy and R. Eberhart, "Particle swarm optimization", in Proceedings of the fourth international Conference on Neural Networks, November/December, 1995, Perth, pp.1942-1948.

[15] S. Mahapatra and M. Singh, " Analysis of symmetrical fault in IEEE 14 bus system for enhancing over current protection scheme", International Journal of Future Generation Communication and Networking, Vol.9, 2016, pp.51-62. 